\title{
DISCerning schizophrenia in mice
}

\section{By C. Simone Fishburn, Senior Editor}

Despite genetic advances in schizophrenia research, a lack of predictive preclinical models has hampered the development of new therapeutics. Now, a team at The Johns Hopkins University has created a transgenic mouse model of prefrontal dysfunction involving disrupted networks of neurons that cause behavioral changes similar to those seen in patients with schizophrenia or mood disorders. ${ }^{1}$

"The mouse here is in a perturbed system based on the genetic model for a neuropsychiatric illness. The model provides for better drug testing than a cell in a dish or an in vivo model that is not a perturbed system."

- Michela Gallagher,

The Johns Hopkins University
The model may represent a useful tool for preclinical characterization of candidate compounds. It centers on the schizophrenia risk marker disrupted in schizophrenia 1 (DISC1) and represents a departure from traditional models that approach the disease as one rooted in an imbalance of neurotransmitters and receptors. Instead, it puts disrupted connectivity in the brain as the cause of schizophrenia.

An inherent problem in employing animal behavioral models in CNS drug development is the difficulty of recapitulating human higher functions such as decision making, emotion and adaptability. Indeed, the vast majority of compounds are evaluated in behavioral assays on normal rats, whose brains do not display the structural and biochemical changes that underlie the different psychoses in humans.

Creating representative models of diseases such as schizophrenia is particularly difficult because of the involvement of multiple genetic factors and environmental triggers. Even when genetic breakthroughs are made, reproducing genetic changes in rats remains technically challenging because of the difficulties of making rat-derived embryonic stem cells despite recent progress in the field. ${ }^{2}$

Michela Gallagher, professor of psychology and neuroscience at Johns Hopkins, and her group at the university have taken a different approach with a model that involves overexpression of an inactive, mutant form of Disc1. ${ }^{1}$

The gene has been strongly linked to schizophrenia predisposition following its identification in a Scottish family that suffered mental illnesses in several generations. ${ }^{3}$ The inactive mutant, a dominant negative form known as DN-DISC1, overrides the action of the regular DISC1 protein, which normally plays a role in the development and maintenance of synapses. Previous studies from other groups have suggested DISC1 is involved in synaptic regulation and the creation of networks that are disrupted in schizophrenia and mood disorders. ${ }^{4}$

"We want to understand how networks of genetically controlled pathways lead to common features of illnesses," Gallagher told SciBX.

She said it is impossible to know whether animals can suffer diseases such as schizophrenia, but what is known is that animal neurons behave electrophysiologically like human ones. She also said there is a large body of literature showing that many high-level systems in the prefrontal cortex are preserved from rodents to primates.

Previously, Gallagher's group generated a heterozygous, transgenic Dn-Disc1 mouse. The animals exhibited detectable biochemical changes in the brain, but behavioral changes were mild. ${ }^{5}$

Now the team has created a homozygous, transgenic Dn-Disc1 mouse. To test higher-level functions of these mice, Gallagher's group focused on activities that require advanced processing of information and that are controlled by the prefrontal cortex.

In a measurement of behavioral flexibility, Dn-Disc1 mice were able to learn new tasks as efficiently as wild-type mice but were not able to adapt readily to a change and took longer to learn the new rules when a task was modified. Likewise, in an assay on outcome expectancy, Dn-Disc1 mice could learn how to perform an action to receive a reward but could not change their actions even when they learned to expect that they would no longer receive the reward.

In tests relating effort to reward, $D n$-Disc1 mice were less motivated than wild-type animals to work hard for a pleasurable reward despite being able to learn the actions needed to receive it. In addition, in social interaction tests, Dn-Disc1 mice were less inclined than wild-type animals to socialize with new mice, and when given a choice between a familiar mouse and a stranger mouse they selected the familiar one. In contrast, wild-type mice preferred the strangers (see Table 1, "Behavior DISCrimination").

The team also observed a 10-fold increase in a marker of oxidative stress in Dn-Disc1 mice specifically in the prefrontal cortex and not in the striatum.

Collectively, the data show that DISC1 affects complex cognitive functions that are controlled by the prefrontal cortex and that DISC1 plays a role in oxidative stress that could underlie damage caused to neurons in that region. The findings also support a role for DISC1 in neuronal networks related to schizophrenia as the altered behavior of the mice mimicked many of those represented in the disorder.

Results were reported in the Proceedings of the National Academy of Sciences.

\section{A failure to communicate}

Gallagher's homozygous Dn-Disc1 model reflects a shift in the CNS field toward the view that schizophrenia and some other CNS disorders are diseases of connectivity, in which a breakdown in communication occurs between neurons and between regions of the brain.

This differs from the view of the disease upon which many current antipsychotics are based, which focused on correcting the over- or underexpression of specific receptors or transmitters.

"The locus of the deficit is not in a particular node but is distributed 
Table 1. Behavior DISCrimination. Experiments are based on fixed-period sessions in which animals are placed in an enclosed chamber, and the number of times they produce a response is counted during that time period. In behavioral flexibility, outcome expectancy and effort/reward assays, the animals are trained in a learning stage to perform an action that yields a food reward. In the test stage, the conditions are changed from their training, and their ability to adapt to the new change is measured.

\begin{tabular}{llll}
\hline Assay & Description & \multicolumn{1}{c}{ Dn-Disc1 mouse } & \multicolumn{1}{c}{ Result } \\
\cline { 2 - 4 } Behavioral flexibility & $\begin{array}{l}\text { Mouse initially learns that pressing the mouse } \\
\text { left lever gives the reward; the sides are } \\
\text { then switched so that the right lever } \\
\text { gives the reward }\end{array}$ & $\begin{array}{l}\text { Adapts to lever switch after multiple } \\
\text { sessions }\end{array}$ & Adapts after one session \\
\hline Outcome expectancy & $\begin{array}{l}\text { Mouse learns to expect that an action } \\
\text { will yield sucrose; the value of sucrose } \\
\text { is then decreased by prefeeding the } \\
\text { animal }\end{array}$ & Maintains interest in the reward & Loses interest in the reward \\
\hline Effort/reward & $\begin{array}{l}\text { Mouse learns to press a lever once for } \\
\text { sucrose; the number of required lever } \\
\text { hits then escalates over time }\end{array}$ & $\begin{array}{l}\text { Loses motivation to hit the lever } \\
\text { multiple times for a reward }\end{array}$ & Sustains motivation \\
\hline Social interaction & $\begin{array}{l}\text { Mouse given a choice of meeting a } \\
\text { stranger mouse or being alone; is } \\
\text { then given a choice of meeting another familiar mouse } \\
\text { new mouse or visiting a familiar } \\
\text { mouse }\end{array}$ & $\begin{array}{l}\text { No preference for meeting a stranger } \\
\text { or being alone, then prefers the }\end{array}$ & Prefers meeting the stranger mouse in \\
both stages of the test
\end{tabular}

around the brain and should be thought of as a network problem. What is important is how one region interacts with other areas of the brain," said Trevor Robbins, professor of cognitive neuroscience at the University of Cambridge.

According to Daniela Brunner, SVP of behavioral R\&D at CNS CRO PsychoGenics Inc., DISC1 is one of the more interesting genes in schizophrenia and other mental disorders. She added that although there are several DISC1 mutants, the dominant negative one, DN-DISC1, is one of the best for understanding the disorder.

Robbins thinks that the new model can be used to make valid inferences about DISC1 and its role in prefrontal dysfunction. "It has been hard to unravel deficits in DISC1 function, but this group has managed to do that," he told SciBX.

One of the key strengths of the Dn-Disc1 model, according to Gallagher, is that the deficits are only in higher-level functions, whereas other systems such as motor control, vision and hearing are not affected. This avoids a significant drawback of many other mutant models in which motor or sensory deficits can cloud the ability to interpret behaviors in tasks that require an animal to move, see or respond to a sound.

According to Brunner, advanced types of behavioral assessments that look at higher cognitive functions are a leap forward from the traditional earlier studies that used simple assays such as the open field test or the Morris water maze.

\section{A low throughput tool}

The translational potential of the model is yet to be determined, but Gallagher believes that the battery of behavioral tests in the Dn-Disc1 background could be better predictors of human behavior than current models.

"The mouse here is in a perturbed system based on the genetic model for a neuropsychiatric illness. The model provides for better drug testing than a cell in a dish or an in vivo model that is not a perturbed system," she told SciBX.

Gallagher cautioned that the model involves an intensive experimental paradigm of complex, multistep behavioral tests and thus is best suited for testing late-stage lead preclinical compounds to help guide clinical studies rather than as a high throughput screening tool.

According to Robbins, one key problem that drug developers have when they employ models of neurological disorders is the failure to take into account behavioral side effects such as memory loss, which can be a major problem for schizophrenia drugs.

Thus, he said the model could have even greater translational utility if it were to include assays for behavioral side effects that involve other regions of the brain, most notably the hippocampus, which plays an important role in memory.

The work has not been patented.

Fishburn, C.S. SciBX 6(33); doi:10.1038/scibx.2013.882 Published online Aug. 29, 2013

\section{REFERENCES}

1. Johnson, A.W. et al. Proc. Natl. Acad. Sci. USA; published online July 9, 2013; doi:10.1073/pnas.1307925110

Contact: Michela Gallagher, The Johns Hopkins University,

Baltimore, Md.

e-mail: michela@jhu.edu

Contact: Akira Sawa, same affiliation as above

e-mail: asawa1@jhmi.edu

2. Tong, C. et al. Nat. Protoc. 6, 827-844 (2011)

3. Millar, J.K. et al. Hum. Mol. Genet. 9, 1415-1423 (2000)

4. Brandon, N.J. \& Sawa, A. Nat. Rev. Neurosci. 12, 707-722 (2011)

5. Hikida, T. et al. Proc. Natl. Acad. Sci. USA 104, 14501-14506 (2007)

\section{COMPANIES AND INSTITUTIONS}

The Johns Hopkins University, Baltimore, Md.

PsychoGenics Inc., Tarrytown, N.Y.

University of Cambridge, Cambridge, U.K. 